\title{
Kaposi sarcoma: an unusual cause of intussusception in an adult patient
}

\author{
OO Jolayemi, ${ }^{1}$ JC Mogambery² \\ ${ }^{1}$ Specialist Surgeon, Ngwelezana Hospital, Empangeni, and Nelson R Mandela School of Medicine, University of \\ KwaZulu-Natal \\ ${ }^{2}$ Physician and Infectious Disease Specialist, Ngwelezana Hospital, Empangeni and Nelson R Mandela School of Medicine, \\ University of KwaZulu-Natal
}

Corresponding author: Olamide Oluwafunsho Jolayemi (funsozn@hotmail.com)

\begin{abstract}
Summary: Kaposi sarcoma (KS) is a multifocal, low-grade tumour of the vascular endothelium and is the most common neoplasm in patients with human immunodeficiency virus and acquired immunodeficiency syndrome (HIV/AIDS). Although $\mathrm{KS}$ is known to cause cutaneous lesions, involvement of the gastrointestinal tract (GIT) is not uncommon. Intussusception secondary to KS however, is exceedingly rare and is a difficult diagnosis to make due to the vague symptomatology. In this case report, we describe the presentation and discuss controversies in the management of such a patient. We also highlight the value of cross sectional imaging in making the diagnosis pre-operatively.
\end{abstract}

S Afr J Surg 2018;56(3) http://dx.doi.org/10.17159/2078-5151/2018/v56n3a2775

\section{Case Presentation}

A 27-year-old man presented to the emergency department with intermittent vomiting and constipation for two weeks, associated with constitutional symptoms of night sweats and weight loss. He denied the presence of respiratory symptoms or contact with any individuals infected with tuberculosis. He had no previous abdominal surgery. He had recently been diagnosed with HIV but was antiretroviral therapy (ART) naïve.

On examination, he had no stigmata of chronic illness. No lesions were present on inspection of his oral cavity or skin.

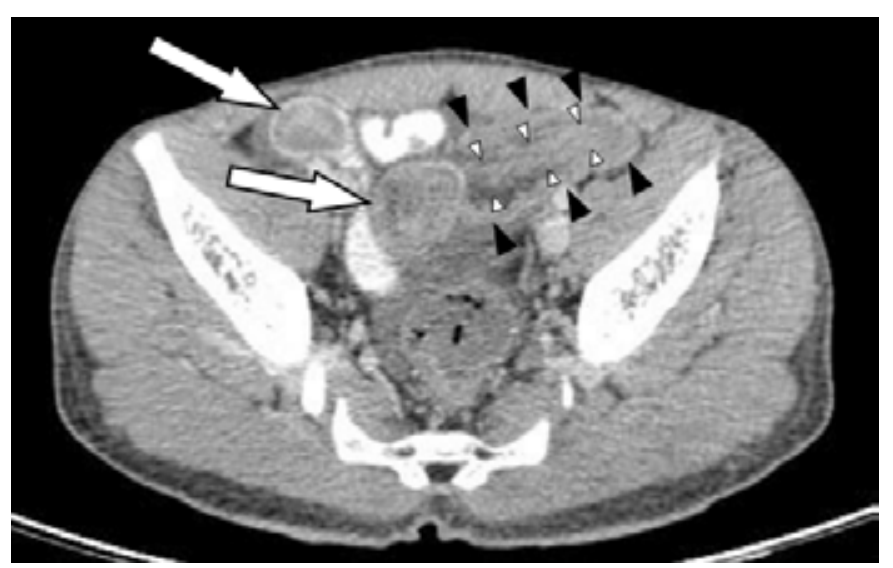

Figure 1a: An axial section demonstrating target signs (white arrows) suggestive of intussusception. Telescoping of proximal bowel (intussuscipiens) into an adjacent distal segment (intussusceptum)) is demonstrated by black triangles and smaller white triangles respectively.

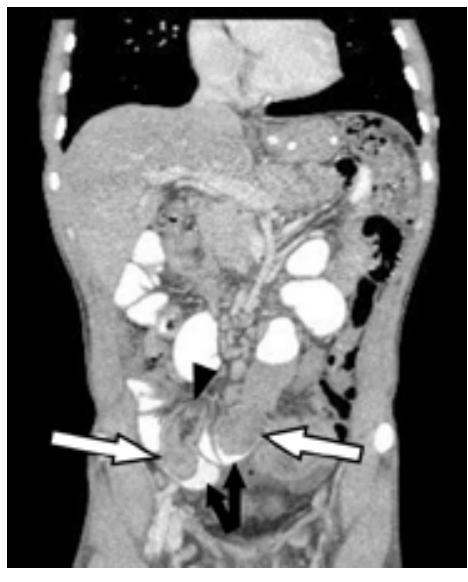

Figure 1b: A coronal section demonstrating intussusceptions (white arrows), with displacement of oral contrast within the intussusceptum (black arrows). Mesenteric fat is depicted by black triangle.

His abdomen was soft but mildly distended, and examination of his hernial orifices revealed no abnormalities. His blood workup, including an arterial blood gas, was unremarkable. Plain radiographs of the chest and abdomen were suggestive of partial small bowel obstruction without perforation. A computed tomography (CT) scan demonstrated multifocal inhomogeneous target signs consisting of small bowel layers, suggestive of intussusception (Figure 1a and b).

The patient underwent an exploratory laparotomy where an ileo-ileal intussusception was found. In addition, there were multiple nodular lesions distributed throughout the entire length of his small bowel. He underwent a resection and primary anastomosis.

Further workup revealed an absolute CD4 count of 28 cells $/ \mu \mathrm{L}$ and HIV viral load of 403000 copies $/ \mathrm{ml}$. He was commenced on ART after the exclusion of opportunistic 
infections. His postoperative course was uneventful and the patient was discharged on the third postoperative day. Histological assessment of the resected segment confirmed a vascular tumour acting as the lead point, with human herpes virus 8 (HHV8) positivity in keeping with KS.

\section{Discussion}

KS was first described in 1872 by Moritz Kaposi, a Hungarian physician and dermatologist. There are three main forms of KS: classical; endemic, which predominantly affects children; and epidemic, which is associated with HIV/AIDS and is the most common variant seen in South Africa. ${ }^{1}$

HHV8 is implicated in the pathogenesis of KS. Once within the host cell, HHV8 expresses various genes that ensure life-long survival through uninhibited cell growth and tumorigenesis. One of the genes, viral gene K13 prevents apoptosis in infected cells once the host cells recognise the virus. Another gene, viral cyclin D, mimics the host cell cyclin $\mathrm{D}$ and is resistant to growth inhibitors allowing cell growth to continue unhindered. Furthermore, the tumour suppressor gene p53 is inhibited by these viral genes and is therefore unable to regulate cell senescence. ${ }^{1}$

Cutaneous $\mathrm{KS}$ is the most common manifestation of the disease. However, the GIT may be involved in up to $6-10 \%$ of patients with AIDS. Lee et al. demonstrated an incidence of $40-51 \%$ for gastrointestinal KS in patients with concomitant cutaneous KS and AIDS. Of these, 12-24\% were distributed in the upper GIT, $8-12 \%$ were in the lower GIT and $15-20 \%$ had multifocal lesions involving both upper and lower GIT. ${ }^{2}$ As with our patient, the absence of cutaneous disease does not preclude involvement of the GIT. In a retrospective review of medical and endoscopy records of $1428 \mathrm{HIV}$-infected patients, Rezende et al. found that $20 \%$ of patients had KS isolated to the GIT. ${ }^{3}$

Intussusception secondary to $\mathrm{KS}$ is a very rare complication. To date, 15 cases have been reported globally with only 7 reported in adults. Moreover, $79 \%$ of patients with GIT KS remain asymptomatic. ${ }^{4}$ Intussusception in adults typically presents with a protracted history of non-specific abdominal symptoms. This vague symptomatology often presents a diagnostic challenge even to the most astute surgeon. Furthermore, in patients with HIV/AIDS, the differentials for gastrointestinal symptomatology is vast. Because surgery is not always indicated, cross-sectional imaging should always be considered to avoid unnecessary surgical intervention in this cohort of patients whose immunological function may predispose them to morbidities from such intervention. The gold standard imaging modality for the diagnosis of intussusception is CT scan. In addition, CT allows for the evaluation of the lead point and for possible complications of bowel obstruction. ${ }^{5}$

In contrast to intussusception in the paediatric population where most cases are idiopathic, adult intussusception is associated with an organic cause acting as the lead point in up to $90 \%$ of cases. Carcinoma accounts for $65-70 \%$ of these therefore surgical resection is universally accepted as the optimal management for adult intussusception. Manual reduction of the involved segment prior to surgical resection however remains a controversial issue. There is consensus that intussusception involving the colon should never be reduced prior to resection due to a higher risk of malignancy. The exception to this is perhaps with sigmoidorectal intussusception in which initial reduction may avert an abdominoperineal resection and a permanent colostomy, but this remains contentious. ${ }^{4}$ We elected to resect the involved segment without reduction in our patient.

Due to its multifocal nature and tendency for recurrence, it is recommended that ART together with chemotherapeutic agents be used as adjuncts to surgery in the management of GIT KS. Studies have shown that ART alone is inferior to ART in combination with chemotherapy for the treatment of KS. ${ }^{1}$. Chemotherapeutic options include the combination of doxorubicin, vincristine and bleomycin or monotherapy with etoposide. These are widely used in South Africa. Monotherapy with lipo-doxorubicin and paclitaxel are also effective and better tolerated although the cost is prohibitive in many South African centers. ${ }^{1,6}$

The patient was referred to the internal medicine and oncology departments at our institution for further treatment. He was commenced on ART during his admission and was responding well to chemotherapy on review.

\section{Conclusion}

Intussusception is a rare complication of $\mathrm{KS}$ and is often difficult to diagnose. Surgeons should have a high index of suspicion in HIV-infected patients who present with protracted non-specific abdominal symptoms. Cross-sectional imaging should be considered in these cases to assist with preoperative planning. The standard of care is surgical resection followed by a combination of ART and chemotherapy.

\section{REFERENCES}

1. Antman K, Chang.Y. Kaposi's Sarcoma. N Engl J Med. 2000;342(14):1027-38. Available from: https://doi.org/10.1056/ nejm200004063421407 [PMID: 10749966]

2. Lee AJ, Brenner L, Mourad B, Monteiro C, et al. Gastrointestinal Kaposi's sarcoma: Case report and review of the literature. J Gastrointest Pharmacol Ther. 6 August 2015;6(3):89-95. Available from: https://doi.org/10.4292/wjgpt.v6.i3.89 [PMCID: PMC4526844]

3. Rezende RE, Kahwage RL, da Costa TV, Machado AA, Brunaldi MO, Kemp R, Modena JL. Upper gastrointestinal Kaposi's sarcoma in HIV-infected patients: ten years of endoscopy observation at a single Brazilian center. Int $\mathrm{J}$ Inf Dis. 2015;39:110-5. Available from: https://doi.org/10.1016/j. ijid.2015.09.006

4. Zubaidi A, Al-Saif F, Silverman R. Adult Intussusception: a Retrospective Review. Dis Colon Rectum. 2006;49(10):1546551. https://doi.org/10.1007/s10350-006-0664-5 [PMID: 16990978]

5. Onkendi EO, Grotz TE, Murray JA, et al. Adult intussusception in the last 25 years of modern imaging: is surgery still indicated? J Gastrointest Surg. 2011;15(10):1699-705. Available from: https://doi.org/10.1007/s11605-011-1609-4 [PMID: 21830152]

6. Gbabe OF, Okwundu CI, Dedicoat M, Freeman EE. Treatment of severe or progressive Kaposi's sarcoma in HIV-infected adults. Cochrane Database Syst Rev. 13 Aug 2014;(8):CD003256. Available from: https://doi.org/10.1002/14651858.cd003256. pub2 [PMID: 25313415] 